TITLE:

\title{
Null controllability with vanishing energy for discrete-time systems
}

$\operatorname{AUTHOR}(\mathrm{S})$ :

Ichikawa, Akira

CITATION:

Ichikawa, Akira. Null controllability with vanishing energy for discretetime systems. Systems \& Control Letters 2008, 57(1): 34-38

ISSUE DATE:

2008-01

URL:

http://hdl.handle.net/2433/84830

\section{RIGHT:}

Copyright (C) 2008 Elsevier B.V.; この論文は出版社版でありません。引 用の際には出版社版をご確認ご利用ください。; This is not the published version. Please cite only the published version. 


\title{
Null Controllability with Vanishing Energy for Discrete-Time Systems
}

\author{
Akira Ichikawa \\ Department of Aeronautics and Astronautics, \\ Kyoto University, Kyoto 606-8501, Japan, \\ Tel: +81757535795 Fax: +81757534975 \\ Email: ichikawa@kuaero.kyoto-u.ac.jp
}

\begin{abstract}
In this paper null controllability with vanishing energy for discrete-time systems is considered. As in the case of continuous time systems necessary and sufficient conditions in terms of an algebraic Riccati equation are given. Then necessary and sufficient conditions involving the eigenvalues of the state matrix are given. Reachability and controllability with vanishing energy are also considered and necessary and sufficient conditions for them are given. Finally applications to sampled-data systems, systems with impulse control and periodic systems are discussed.
\end{abstract}

Keywords: discrete-time systems, null controllability, optimal regulator, Riccati equation

\section{Introduction}

The relative motion of a spacecraft with respect to another in a circular orbit around the Earth is described by autonomous nonlinear differential equations. The linearized equations are known as Hill's equations [7]. The state matrix of their state space representation has all its eigenvalues on the imaginary axis. The motion in the orbit plane (the in-plane motion) is independent of the out-of-plane motion and a controlled version has the form

$$
\dot{x}=\left[\begin{array}{cccc}
0 & 0 & 1 & 0 \\
0 & 0 & 0 & 1 \\
3 \omega^{2} & 0 & 0 & 2 \omega \\
0 & 0 & -2 \omega & 0
\end{array}\right] x+\left[\begin{array}{ll}
0 & 0 \\
0 & 0 \\
1 & 0 \\
0 & 1
\end{array}\right] u
$$

where $\omega$ is the orbit rate (angular velocity) of the satellite in a circular motion.

The system (1) is controllable and any state can be steered to the origin with a control of an arbitrarily small $L_{2}$-norm. This property is called the null controllability with vanishing energy [5]. If the origin is replaced by an arbitrary final state, a stronger property, called controllability with vanishing energy, is obtained. The system (1) also has this property. These notions are introduced originally for infinite dimensional systems [5]. But they are no less important in finite dimensions and their main results can be described as follows. Consider the linear system

$$
\dot{x}=A x+B u,
$$

where $x \in R^{n}$ and $u \in R^{m}$.

Let $x\left(t ; x_{0}, u\right)$ be the solution of $(2)$ with initial condition $x(0)=x_{0}$. We denote by $|\cdot|$ the Euclidean norm of vectors and by $\sigma(A)$ the the set of all eigenvalues of $A$.

Definition 1.1 (a) The system (2) is said to be null controllable with vanishing energy (NCVE for short) if for each initial $x(0)=x_{0}$ there exists a sequence of pairs $\left(T_{N}, u_{N}\right), 0<T_{N} \uparrow \infty, u_{N} \in L_{2}\left(0, T_{N} ; R^{m}\right)$ such that $x\left(T_{N} ; x_{0}, u_{N}\right)=0$ and

$$
\lim _{N \rightarrow \infty} \int_{0}^{T_{N}}\left|u_{N}(t)\right|^{2} d t=0 .
$$


(b) The system (2) is said to be controllable with vanishing energy (CVE) if for any pair $\left(x_{0}, x_{1}\right)$ of initial and final states there exists a sequence of pairs $\left(T_{N}, u_{N}\right), 0<T_{N} \uparrow \infty, u_{N} \in L_{2}\left(0, T_{N} ; R^{m}\right)$ such that $x\left(T_{N} ; x_{0}, u_{N}\right)=x_{1}$ and (3) holds.

$(A, B)$ is said to be NCVE (CVE) if the system (2) is NCVE (CVE). Necessary and sufficient conditions for NCVE and CVE are given as follows [5].

Theorem $1.1(A, B)$ is $N C V E$ if and only if

(a) it is controllable, and

(b) $X=0$ is the unique solution of the algebraic Riccati equation (ARE)

$A^{\prime} X+X A-X B B^{\prime} X=0$

in the class of nonnegative matrices.

Theorem $1.2(A, B)$ is $N C V E$ if and only if

(a) $(A, B)$ is controllable, and

(b) $\operatorname{Re}(\lambda) \leq 0$ for any $\lambda \in \sigma(A)$.

Theorem $1.3(A, B)$ is $C V E$ if and only if

(a) $(A, B)$ is controllable, and

(b) $\operatorname{Re}(\lambda)=0$ for any $\lambda \in \sigma(A)$.

As we can see from theorems above, controllable mass-spring systems are CVE and hence NCVE and electric circuits are NCVE if they are controllable. The proof of Theorem 1.1 is based on the theory of optimal quadratic control. For the proof of necessity of Theorem 1.2 the relation between the Riccati equation and the controllability gramian of the pair $(-A, B)$ is used, while for sufficiency the Riccati equation is used. Theorem 1.3 is a consequence of Theorem 1.2 and the fact that $(-A,-B)$ is also NCVE.

In this paper we shall consider the discrete-time versions of these theorems. It is important in its own right but also useful when we consider sampled-data systems with zero-order hold, systems with impulse control and periodic systems as discussed in Section 5. In fact this paper is motivated by the extension of NCVE to the generalized Hill's equations where the target's orbit is elliptic and the system (1) is replaced by a periodic system [6]. In the discrete-time case some new features arise in the developments. For example the proof of necessity of Theorem 1.2 becomes more involved since the Riccati equation is more complicated for discrete-time systems. Lemma 2.5 in Section 2 fills this gap and enables us to extend the theorem. The extension of Theorem 1.3 requires the invertibility of $A$ which is not used for continuous time systems.

This paper is organized as follows. In Section 2 we give preliminaries concerning necessary notions of discrete-time systems. In Section 3 we consider necessary and sufficient conditions for NCVE and extend Theorem 1.1 and Theorem 1.2. In Section 4 we introduce reachability with vanishing energy and extend Theorem 1.3. Finally in Section 5 we apply NCVE and CVE results to sampled-data systems, systems with impulse control and periodic systems. In particular, we show that sampled-data systems are NCVE if and only if the underlining continuous time systems are NCVE, provided the sampling periods are nonpathological. NCVE and CVE of the generalized Hill's equation are shown in [6].

\section{Preliminaries}

Consider the discrete-time system

$$
x(k+1)=A x(k)+B u(k),
$$

where $x \in R^{n}$ and $u \in R^{m}$. We recall from [1] basic definitions and some useful results for (5).

Definition 2.1 (a) The state $x_{0}$ is controllable to zero on $\left[0, n_{0}\right]$ if there exists a sequence of control inputs $u=\left\{u(0), u(1), \ldots, u\left(n_{0}-1\right)\right\}$ such that $x\left(n_{0} ; x_{0}, u\right)=0$.

(b) The state $x_{1}$ is reachable (from zero) on $\left[0, n_{1}\right]$ if there exists a sequence of control inputs $u=\left\{u(0), u(1), \ldots, u\left(n_{1}-1\right)\right\}$ such that $x\left(n_{1} ; 0, u\right)=x_{1}$.

(c) $(A, B)$ is null controllable on $\left[0, n_{0}\right]$ if every state is controllable to zero on $\left[0, n_{0}\right]$. 
(d) $(A, B)$ is reachable $\left[0, n_{1}\right]$ if every state is reachable on $\left[0, n_{1}\right]$.

(e) $(A, B)$ is controllable on $\left[0, n_{2}\right]$ if for every pair $\left(x_{0}, x_{1}\right)$ there is a a sequence of control inputs $u=\left\{u(0), u(1), \ldots, u\left(n_{2}-1\right)\right\}$ such that $x\left(n_{2} ; x_{0}, u\right)=x_{1}$.

Lemma 2.1 (a) The state $x_{0}$ is controllable to zero on $\left[0, n_{0}\right]$, for some $n_{0}>0$ if and only if it is controllable to zero on $[0, n]$, where $n$ is the dimension of the system (5). In this case $x_{0}$ is controllable to zero on $[0, N]$ for any $N \geq n_{0}$.

(b) The state $x_{1}$ is reachable on $\left[0, n_{1}\right]$, for some $n_{1}>0$ if and only if it is reachable on $[0, n]$. In this case $x_{1}$ is reachable on $[0, N]$ for any $N \geq n_{1}$.

Definition $2.2(A, B)$ is null controllable, reachable and controllable respectively if it is null controllable, reachable and controllable on $[0, n]$ respectively.

Lemma 2.2 (a) $(A, B)$ is reachable if and only if it is controllable. In this case it is null controllable. (b) Suppose $A$ is nonsingular. Then $(A, B)$ is null controllable if and only if it is controllable.

Lemma 2.3 The following statements are equivalent.

(a) $(A, B)$ is null controllable.

(b) For any $x_{0}, A^{n} x_{0} \in R\left(M_{c}\right)$, where $M_{c}=\left[B A B \ldots A^{n-1} B\right]$ is the reachability matrix.

(c) $\operatorname{rank}[\lambda I-A B]=n$ for any $0 \neq \lambda \in \sigma(A)$.

Lemma 2.4 Suppose $A$ is stable i.e., $|\lambda|<1$ for any $\lambda \in \sigma(A)$ and that $(A, B)$ is controllable. Then there exists a positive definite matrix $Y$ satisfying

$$
Y=A Y A^{\prime}+B B^{\prime}
$$

$Y$ is called the controllability gramian of $(A, B)$.

Lemma 2.5 Suppose $A$ is antistable i.e., $|\lambda|>1$ for any $\lambda \in \sigma(A)$ and that $(A, B)$ is controllable. Then $A^{-1}$ is stable and $\left(A^{-1}, A^{-1} B\right)$ is controllable. The inverse of its controllability gramian $Y$ satisfies the following algebraic Riccati equation

$$
X=A^{\prime} X A-A^{\prime} X B\left(I+B^{\prime} X B\right)^{-1} B^{\prime} X A .
$$

Proof. Since $A$ is antistable, it is nonsingular. Since $(A, B)$ is controllable, so is $\left(A^{-1}, A^{-1} B\right)$. Now by definition

$$
Y=A^{-1} Y\left(A^{-1}\right)^{\prime}+A^{-1} B B^{\prime}\left(A^{-1}\right)^{\prime},
$$

which implies

$$
A Y A^{\prime}=Y+B B^{\prime}
$$

This in turn yields see Lemma 3.18 [4]

$$
\begin{aligned}
\left(A^{-1}\right)^{\prime} Y^{-1} A^{-1} & =\left(Y+B B^{\prime}\right)^{-1} \\
& =Y^{-1}\left(I+B B^{\prime} Y^{-1}\right)^{-1} \\
& =Y^{-1}\left[I-\left(I+B B^{\prime} Y^{-1}\right)^{-1} B B^{\prime} Y^{-1}\right] \\
& =Y^{-1}\left[I-B\left(I+B^{\prime} Y^{-1} B\right)^{-1} B^{\prime} Y^{-1}\right] \\
& =Y^{-1}-Y^{-1} B\left(I+B^{\prime} Y^{-1} B\right)^{-1} B^{\prime} Y^{-1},
\end{aligned}
$$

where for the fourth equality we have used the familiar identity $M(I+N M)^{-1}=(I+M N)^{-1} M$. Hence we obtain

$$
Y^{-1}=A^{\prime} Y^{-1} A-A^{\prime} Y^{-1} B\left(I+B^{\prime} Y^{-1} B\right)^{-1} B^{\prime} Y^{-1} A
$$

and $Y^{-1}$ is a positive solution of the ARE (6). 


\section{Null controllability with vanishing energy}

Consider the system (5)

$$
x(k+1)=A x(k)+B u(k) .
$$

We shall define NCVE for this system.

Definition $3.1(A, B)$ is null controllable with vanishing energy if for each $x_{0}$ there exists a sequence of pairs $\left(k_{N}, u_{N}\right), k_{N}$ a positive integer $\uparrow \infty, u_{N} \in l_{2}\left(0, k_{N}-1 ; R^{m}\right)$ such that $x\left(k_{N} ; x_{0}, u_{N}\right)=0$ and

$$
\lim _{N \rightarrow \infty}\left\|u_{N}\right\|_{2}=0
$$

where $l_{2}\left(0, k_{N}-1 ; R^{m}\right)$ is the set of vectors $u=\left\{u(0), u(1), \ldots, u\left(k_{N}-1\right)\right\}, u(k) \in R^{m}$ with norm

$$
\|u\|_{2}=\left(\sum_{k=0}^{k_{N}-1}|u(k)|^{2}\right)^{\frac{1}{2}} \text {. }
$$

We shall prove the following.

Theorem $3.1(A, B)$ is NCVE if and only if

(a) $(A, B)$ is null controllable, and

(b) $X=0$ is the unique solution of the ARE (6)

$$
X=A^{\prime} X A-A^{\prime} X B\left(I+B^{\prime} X B\right)^{-1} B^{\prime} X A
$$

in the class of nonnegative matrices.

Theorem $3.2(A, B)$ is $N C V E$ if and only if

(a) $(A, B)$ is null controllable, and

(b) $|\lambda| \leq 1$ for any $\lambda \in \sigma(A)$.

Proof of Theorem 3.1.

We first show necessity. Consider the quadratic cost associated with $(5)$ on $\left[0, k_{N}-1\right]$

$$
J\left(u ; x_{0}, k_{N}, Q\right)=\sum_{k=0}^{k_{N}-1}|u(k)|^{2}+x\left(k_{N}\right)^{\prime} Q x\left(k_{N}\right),
$$

where $Q \geq 0$. It is known [1], [4] that the optimal control minimizing the cost function is given by the feedback law

$$
\bar{u}(k)=-\left[I+B^{\prime} X(k+1) B\right]^{-1} B^{\prime} X(k+1) A x(k),
$$

where $X(k)=X\left(k ; k_{N}, Q\right)$ is the sequence of nonnegative matrices defined by the Riccati equation

$$
\begin{aligned}
X(k) & =A^{\prime} X(k+1) A-A^{\prime} X(k+1) B\left[I+B^{\prime} X(k+1) B\right]^{-1} B^{\prime} X(k+1) A, \\
X\left(k_{N}\right) & =Q .
\end{aligned}
$$

Moreover,

$$
J\left(\bar{u} ; x_{0}, k_{N}, Q\right)=x_{0}^{\prime} X\left(0 ; k_{N}, Q\right) x_{0} .
$$

Now we consider the case $Q=q I, q>0$ and let $q \rightarrow \infty$. Since $(A, B)$ is null controllable, for each $x_{0}$ and $k_{N} \geq n$ there exists a control $u \in l_{2}\left(0, k_{N}-1 ; R^{m}\right)$ such that $x\left(k_{N} ; x_{0}, u\right)=0$. Let $u_{N}$ be the control with minimum norm among them. Then it is given by $u_{N}=-M_{N}^{+} A^{k_{N}} x_{0}$ where $M_{N}^{+}=M_{N}^{\prime}\left(M_{N} M_{N}^{\prime}\right)^{-1}$ and $M_{N}=\left[A^{k_{N}-1} B \ldots A B B\right]$. Since $(A, B)$ is NCVE, $\lim _{N \rightarrow \infty}\left\|u_{N}\right\|_{2}^{2}=\lim _{N \rightarrow \infty}\left|M_{N}^{+} A^{k_{N}} x_{0}\right|^{2}=0$ for each $x_{0}$. Hence there exists a constant $a>0$ such that $\left\|u_{N}\right\|_{2}^{2} \leq a\left|x_{0}\right|^{2}$. Notice that

$$
J\left(\bar{u} ; x_{0}, k_{N}, q I\right)=x_{0}^{\prime} X\left(0 ; k_{N}, q I\right) x_{0} \leq J\left(u_{N} ; x_{0}, k_{N}, q I\right)=\left\|u_{N}\right\|_{2}^{2} \leq a\left|x_{0}\right|^{2},
$$


which yields $X\left(0 ; k_{N}, q I\right) \leq a I$. Since $X\left(0 ; k_{N}, q I\right)$ is monotone increasing in $q$, there exists a limit as $q \rightarrow \infty$, denoted by $X\left(0 ; k_{N}\right)$, i.e., $X\left(0 ; k_{N}\right)=\lim _{q \rightarrow \infty} X\left(0 ; k_{N}, q I\right)$. Let $\bar{u}_{q}$ be the optimal control for $J\left(u ; x_{0}, k_{N}, q I\right)$. Then it is uniformly bounded in $q$. Hence there exists a subsequence $q_{j}$ such that $\bar{u}_{q_{j}}$ converges to some limit $\bar{u}_{\infty}$. Then $x\left(k_{N} ; x_{0}, \bar{u}_{\infty}\right)=0$ and $\left\|u_{\infty}\right\|_{2}^{2}=x_{0}^{\prime} X\left(0 ; k_{N}\right) x_{0} \leq\left\|u_{N}\right\|_{2}^{2}$. Hence $x_{0}^{\prime} X\left(0 ; k_{N}\right) x_{0}=\left\|u_{N}\right\|_{2}^{2}$. Now suppose that $(A, B)$ is null controllable on $\left[0, n_{0}\right], n_{0} \leq n$. Since $X\left(k ; k_{N}, q I\right)=X\left(0 ; k_{N}-k, q I\right)$, the following limit exists:

$$
\lim _{q \rightarrow \infty} X\left(k ; k_{N}, q I\right)=\lim _{q \rightarrow \infty} X\left(0 ; k_{N}-k, q I\right) \equiv X\left(k ; k_{N}\right) \text { for } k \leq k_{N}-n_{0} .
$$

Moreover, from equation (9) $X\left(k ; k_{N}\right), k \leq k_{N}-n_{0}$ satisfies the Riccati equation

$$
\begin{aligned}
X(k) & =A^{\prime} X(k+1) A-A^{\prime} X(k+1) B\left[I+B^{\prime} X(k+1) B\right]^{-1} B^{\prime} X(k+1) A, \\
X\left(k_{N}-n_{0}\right) & =X\left(k_{N}-n_{0} ; k_{N}\right) .
\end{aligned}
$$

Since $x_{0}^{\prime} X\left(0 ; k_{N}\right) x_{0}=\left\|u_{N}\right\|_{2}^{2}, X\left(0 ; k_{N}\right)$ is decreasing in $N$ and has a nonnegative limit

$$
X_{\infty}=\lim _{N \rightarrow \infty} X\left(0 ; k_{N}\right) \text {. }
$$

For $k \leq N-n_{0}$ we know $X\left(k ; k_{N}\right)=X\left(0 ; k_{N}-k\right)$ and hence $\lim _{N \rightarrow \infty} X\left(k ; k_{N}\right)=X_{\infty}$. Letting $N \rightarrow \infty$ in the Riccati equation above we see that $X_{\infty}$ satisfies the $\operatorname{ARE}(6)$. Recall that $(A, B)$ is NCVE and hence

$$
x_{0}^{\prime} X_{\infty} x_{0} \leq x_{0}^{\prime} X\left(0 ; k_{N}\right) x_{0}=\left\|u_{N}\right\|_{2}^{2} \rightarrow 0
$$

and $X_{\infty}=0$. Now let $X$ be any nonnegative solution of the ARE (6). We shall show that $X \leq X_{\infty}$ to conclude $X=0$. For this purpose consider the Riccati difference equation (9) with $Q=X$. Then $X(k)=X$ is a solution. Thus

$$
J\left(\bar{u} ; x_{0}, k_{N}, X\right)=x_{0}^{\prime} X x_{0} \leq J\left(\bar{u} ; x_{0}, k_{N}, q I\right)=x_{0}^{\prime} X\left(0 ; k_{N}, q I\right) x_{0}
$$

for large $q$, where $\bar{u}$ denotes the optimal control for each cost function. Now passing to the limit $q \rightarrow \infty$ and to the limit $N \rightarrow \infty$ we obtain $x_{0}^{\prime} X x_{0} \leq x_{0}^{\prime} X\left(0 ; k_{N}\right) x_{0}$ and $x_{0}^{\prime} X x_{0} \leq x_{0}^{\prime} X_{\infty} x_{0}$ respectively. Thus we have shown $X=0$, which completes the proof of necessity.

To show sufficiency we recall that $\left\|u_{N}\right\|_{2}^{2}=x_{0}^{\prime} X\left(0 ; k_{N}\right) x_{0} \rightarrow x_{0}^{\prime} X_{\infty} x_{0}$. But by condition (b) $X_{\infty}=0$ and hence $\left\|u_{N}\right\|_{2} \rightarrow 0$ and $(A, B)$ is NCVE.

Proof of Theorem 3.2

To show necessity we suppose that there exists an eigenvalue $\lambda$ with $|\lambda|>1$. Considering the eigenspaces of $A$ we can find a nonsingular matrix $T$ such that $\tilde{x}=T x$ yields

$$
\tilde{x}(k+1)=\left[\begin{array}{cc}
\tilde{A}_{1} & 0 \\
0 & \tilde{A}_{2}
\end{array}\right] \tilde{x}(k)+\left[\begin{array}{c}
\tilde{B}_{1} \\
\tilde{B}_{2}
\end{array}\right] u(k) \equiv \tilde{A} \tilde{x}(k)+\tilde{B} u(k),
$$

where $|\lambda|>1$ for any $\lambda \in \sigma\left(\tilde{A}_{1}\right)$ and $|\lambda| \leq 1$ for any $\lambda \in \sigma\left(\tilde{A}_{2}\right)$. Notice that $\left(\tilde{A}_{1}, \tilde{B}_{1}\right)$ and $\left(\tilde{A}_{2}, \tilde{B}_{2}\right)$ are NCVE. Since $\tilde{A}_{1}$ is antistable, it is nonsingular and $\left(\tilde{A}_{1}, \tilde{B}_{1}\right)$ is controllable by Lemma 2.2. Moreover, $\left(\tilde{A}_{1}^{-1}, \tilde{A}_{1}^{-1} \tilde{B}_{1}\right)$ is controllable. Hence by Lemma 2.4 it possesses a positive controllability gramian $Y$

$$
Y=\tilde{A}_{1}^{-1} Y\left(\tilde{A}_{1}^{-1}\right)^{\prime}+\tilde{A}_{1}^{-1} \tilde{B}_{1} \tilde{B}_{1}^{\prime}\left(\tilde{A}_{1}^{-1}\right)^{\prime} .
$$

By Lemma $2.5 X_{1}=Y^{-1}$ is a positive solution of the ARE

$$
X=\tilde{A}_{1}^{\prime} X \tilde{A}_{1}-\tilde{A}_{1}^{\prime} X \tilde{B}_{1}\left(I+\tilde{B}_{1}^{\prime} X \tilde{B}_{1}\right)^{-1} \tilde{B}_{1}^{\prime} X \tilde{A}_{1} .
$$

Then $\tilde{X}=\left[\begin{array}{cc}\tilde{X}_{1} & 0 \\ 0 & 0\end{array}\right]$ is a nontrivial nonnegative solution of the $\operatorname{ARE}$ for $(\tilde{A}, \tilde{B})$

$$
\tilde{X}=\tilde{A}^{\prime} \tilde{X} \tilde{A}-\tilde{A}^{\prime} \tilde{X} \tilde{B}\left(I+\tilde{B}^{\prime} \tilde{X} \tilde{B}\right)^{-1} \tilde{B}^{\prime} \tilde{X} \tilde{A},
$$

which contradicts to Theorem 3.1. Hence $|\lambda| \leq 1$ for any $\lambda \in \sigma(A)$.

To show sufficiency let $X$ be any nonnegative solution of the ARE (6). Let $\lambda \in \sigma(A)$ with a corresponding eigenvector $p$ i.e., $|\lambda| \leq 1, A p=\lambda p$. Then

$$
\begin{aligned}
p^{*} X p & =p^{*} A^{\prime} X A p-p^{*} A^{\prime} X B\left(I+B^{\prime} X B\right)^{-1} B^{\prime} X A p \\
& =|\lambda|^{2}\left[p^{*} X p-p^{*} X B\left(I+B^{\prime} X B\right)^{-1} B^{\prime} X p\right],
\end{aligned}
$$


where $p^{*}$ is the Hermitian transpose of $p$. If $|\lambda|<1$, then from (10) we conclude $X p=0$. Now suppose $|\lambda|=1$. Then (10) yields $B^{\prime} X p=0$. Again from the ARE (6) we obtain $X p=\lambda A^{\prime} X p$. Hence $p^{*} X\left[\frac{1}{\lambda} I-A B\right]=0$. By Lemma 2.3 we conclude $X p=0$. Hence for any eigenvector of $A$ we have shown $X p=0$. We shall show that $X q=0$ for any generalized eigenvector of $A$, which concludes $X=0$. Now let $q \in N\left((\lambda I-A)^{2}\right)$ i.e., $(\lambda I-A)^{2} q=0$. Then $q_{1}=(\lambda I-A) q$ satisfies $(\lambda I-A) q_{1}=0$. Repeating the arguments above we conclude $X q_{1}=0$. Hence $X A q=\lambda X q$ and from the ARE (6) we obtain

$$
\begin{aligned}
q^{*} X q & =q^{*} A^{\prime} X A q-q^{*} A^{\prime} X B\left(I+B^{\prime} X B\right)^{-1} B^{\prime} X A q \\
& =|\lambda|^{2}\left[q^{*} X q-q^{*} X B\left(I+B^{\prime} X B\right)^{-1} B^{\prime} X q\right] .
\end{aligned}
$$

This is the same with $(10)$ and hence $X q=0$. Repeating this process we conclude $X q=0$ for any generalized eigenvector of $A$ satisfying $(\lambda I-A)^{k} q=0$. Hence $X=0$ and by Theorem $3.1(A, B)$ is NCVE.

\section{Controllability with vanishing energy}

First we introduce reachability with vanishing energy (RVE), which is useful when we study CVE.

Definition $4.1(A, B)$ is reachable with vanishing energy if for each $x_{1}$ there exists a sequence of pairs $\left(k_{N}, u_{N}\right), k_{N} \uparrow \infty, u_{N} \in l_{2}\left(0, k_{N}-1 ; R^{m}\right)$ such that $x\left(k_{N} ; 0, u_{N}\right)=x_{1}$ and

$$
\lim _{N \rightarrow \infty}\left\|u_{N}\right\|_{2}=0
$$

Lemma 4.1 Suppose $(A, B)$ is RVE. Then $A$ is nonsingular. Moreover, $(A, B)$ is RVE if and only if $\left(A^{-1}, A^{-1} B\right)$ is NCVE.

Proof. Suppose $A$ is nilpotent so that $A^{j}=0$ for any $j \geq n$. Let $x_{1}$ be arbitrary. Then for any $N \geq n$ there is a control $u_{N}$ such that

$$
\sum_{k=0}^{N-1} A^{N-1-k} B u_{N}(k)=\sum_{k=N-n}^{N-1} A^{N-1-k} B u_{N}(k)=\sum_{j=0}^{n-1} A^{j} B u_{N}(N-1-j)=x_{1} .
$$

Since the last equation is independent of $N$ and involves only $n$-unknown input vectors, $\left\|u_{N}\right\|_{2}$ cannot converge to zero and RVE of $(A, B)$ is impossible. Now suppose $A$ is singular. Then $(A, B)$ can be transformed to an equivalent $(\tilde{A}, \tilde{B})$ with

$$
\tilde{A}=\left[\begin{array}{cc}
A_{0} & 0 \\
0 & A_{1}
\end{array}\right], \tilde{B}=\left[\begin{array}{l}
B_{0} \\
B_{1}
\end{array}\right]
$$

such that $A_{0}$ is nilpotent, $A_{1}$ nonsingular and $\left(A_{0}, B_{0}\right)$ is RVE. However the last property contradicts what we have shown above. Hence $A$ is nonsingular. Now when $A$ is nonsingular, the system (5) can be written as

$$
x(k)=A^{-1} x(k+1)-A^{-1} B u(k) .
$$

Thus if $(A, B)$ is RVE, then redefining $u$ and $x$ we can easily see that

$$
\tilde{x}(k+1)=A^{-1} \tilde{x}(k)+A^{-1} B \tilde{u}(k) .
$$

is NCVE. The converse is also true since we can reverse the arguments.

\section{Remark 4.1 Consider the pair}

$$
A=\left[\begin{array}{ll}
0 & 1 \\
0 & 0
\end{array}\right], B=\left[\begin{array}{l}
0 \\
1
\end{array}\right] .
$$

Then $(A, B)$ is controllable and $A$ is nilpotent. We can directly obtain $\left\|u_{N}\right\|_{2}=\left|x_{1}\right|$, where $u_{N}$ is the control with minimum norm such that $x\left(k_{N} ; 0, u_{N}\right)=x_{1}$. Hence $(A, B)$ is not RVE.

From Lemma 4.1 we immediately obtain the following. 
Theorem $4.1(A, B)$ is RVE if and only if

(a) $(A, B)$ is controllable, and

(b) $|\lambda| \geq 1$ for any $\lambda \in \sigma(A)$.

Now we are ready to extend Theorem 1.3.

Theorem $4.2(A, B)$ is $C V E$ if and only if

(a) $(A, B)$ is controllable, and

(b) $|\lambda|=1$ for any $\lambda \in \sigma(A)$.

Proof. Note that $(A, B)$ is CVE if and only if it is NCVE and RVE. Hence the proof follows from Theorem 3.2 and Theorem 4.2 .

\section{Applications}

In this section we apply our theorems to sampled-data systems, systems with impulse control and periodic systems. First we consider a sampled-data system with zero-order hold

$$
\dot{x}=A x+B u,
$$

where

$$
u(t)=u(k \tau), k \tau \leq t<(k+1) \tau .
$$

Then at times $k \tau$ we have the following.

$$
\begin{aligned}
x((k+1) \tau) & =\exp [\tau A] x(k \tau)+\int_{0}^{\tau} \exp [r A] B d r u(k \tau) \\
& \equiv A_{d} x(k \tau)+B_{d} u(k \tau) .
\end{aligned}
$$

The sampled-data system above is said to be NCVE (CVE) if it is NCVE (CVE) in the sense of Definition 1.1 with $T_{N}=N \tau$. Obviously the sampled-data system is NCVE (CVE) if and only if $\left(A_{d}, B_{d}\right)$ is NCVE (CVE). But by Theorems 3.2 and $4.2\left(A_{d}, B_{d}\right)$ is NCVE (CVE) if and only if

(a) $\left(A_{d}, B_{d}\right)$ is controllable, and

(b) $|\lambda| \leq 1(|\lambda|=1)$ for any $\lambda \in \sigma\left(A_{d}\right)$.

If the sampling period $\tau$ is nonpathological [2], i.e., there is no pair $\left(\lambda_{1}, \lambda_{2}\right)$ of distinct eigenvalues of $A$ such that $\left|\operatorname{Im}\left(\lambda_{1}\right)-\operatorname{Im}\left(\lambda_{2}\right)\right|=k \frac{2 \pi}{\tau}$ for some positive integer $k$, then the controllability of $(A, B)$ implies (a). The condition (b) holds if and only if $\operatorname{Re}(\lambda) \leq 0(\operatorname{Re}(\lambda)=0)$ for any $\lambda \in \sigma(A)$. Hence we obtain the following.

Theorem 5.1 Suppose that the sampling period $\tau$ is nonpathological. Then the sampled-data system is NCVE (CVE) if and only if $(A, B)$ is NCVE (CVE).

Next we consider the system (2) with impulse control $u(k-1) \delta(t-k \tau)$ at time $k \tau, k \geq 1$. Then the state $x(k \tau)$ after the impulse $u(k-1) \delta(t-k \tau)$ satisfies

$$
x((k+1) \tau)=\exp [\tau A] x(k \tau)+B u(k) .
$$

Moreover, the system with impulse control is NCVE (CVE) if and only if (if) (exp $[k \tau A], B)$ is NCVE (CVE). Hence we have the following.

Theorem 5.2 The the system (2) with impulse control is NCVE (CVE) if $(A, B)$ is NCVE (CVE) and $(\exp [k \tau A], B)$ is controllable.

Finally consider the $T$-periodic system

$$
\dot{x}=A(t) x+B(t) u,
$$

where $A(t)$ and $B(t)$ are $T$-periodic continuous matrices. Let $S(t, s)$ be the transition matrix associated with $A(t)$. Then

$$
\begin{aligned}
x((k+1) T) & =S(T, 0) x(k T)+\int_{0}^{T} S(T, r) B(r) u(k, r) d r \\
& \equiv S(T, 0) x(k T)+B_{d} u(k),
\end{aligned}
$$


where $u(k, r)=u(k T+r)$, for $0 \leq r<T, u(k)=u(k, \cdot) \in L_{2}\left(0, T ; R^{m}\right)$ and $B_{d}$ is a bounded linear operator in $\mathcal{L}\left(L_{2}\left(0, T ; R^{m}\right), R^{n}\right)$. Notice that the periodic system is NCVE (CVE) if and only if (if) $\left(S(T, 0), B_{d}\right)$ is NCVE $(\mathrm{CVE})$. Recall that $\left(S(T, 0), B_{d}\right)$ is controllable if

$$
M_{T}=\int_{0}^{T} S(T, r) B(r) B^{\prime}(r) S^{\prime}(T, r) d r>0 .
$$

Now by a modification of Theorem 3.2 and Theorem 4.2 we obtain the following.

Theorem 5.3 The periodic system is $N C V E(C V E)$ if $|\lambda| \leq 1(|\lambda|=1)$ for any $\lambda \in \sigma(S(T, 0))$ and $M_{T}>0$.

Using this theorem NCVE and CVE of the generalized Hill's equation are shown in [6]. The control space for the discrete-time system above is infinite dimensional and $B_{d}$ is an operator rather than a matrix. Full treatment of NCVE for discrete-time systems in infinite dimensions will be given elsewhere. NCVE of infinite dimensional periodic systems is considered in [3].

\section{References}

[1] F. M. Callier and C. A. Desoer, Linear System Theory, Springer-Verlag, Berlin, 1991.

[2] T. Chen and B. A. Francis, Optimal Sampled-Data Control Systems, Springer-Verlag, London, 1995.

[3] A. Ichikawa, Null controllability with vanishing energy for infinite dimensional periodic systems, Proc. 17th International Symposium on Mathematical Theory of Networks and Systems, July, 2006, $638-642$.

[4] A. Ichikawa and H. Katayama, Linear Time Varying Systems and Sampled-data Systems, SpringerVerlag, London, 2001.

[5] E. Priola and J. Zabczyk, Null controllability with vanishing energy, SIAM J. Control Optim., 42 (2003), 1013-1032.

[6] M. Shibata and A. Ichikawa, Orbital rendezvous and flyaround based on null controllability with vanishing energy, Journal of Guidance, Control, and Dynamics, to appear.

[7] B. Wie, Space Vehicle Dynamics and Control, American Institute of Aeronautics and Astronautics, 1998. 